\title{
Methylglyoxal increases cardiomyocyte ischemia-reperfusion injury via glycative inhibition of thioredoxin activity.
}

Xiaoliang Wang

Thomas Jefferson University

Wayne B. Lau

Thomas Jefferson University

Yue-Xing Yuan

Thomas Jefferson University

Ya-Jing Wang

Thomas Jefferson University

HoibW this and additional works at: https://jdc.jefferson.edu/emfp Thomas Jefferson University

Part of the Emergency Medicine Commons

\section{Let us know how access to this document benefits you}

\section{Recommended Citation}

Wang, Xiaoliang; Lau, Wayne B.; Yuan, Yue-Xing; Wang, Ya-Jing; Yi, Wei; Christopher, Theodore A.; Lopez, Bernard L.; Liu, Hui-Rong; and Ma, Xin-Liang, "Methylglyoxal increases cardiomyocyte ischemia-reperfusion injury via glycative inhibition of thioredoxin activity." (2010). Department of Emergency Medicine Faculty Papers. Paper 156.

https://jdc.jefferson.edu/emfp/156

This Article is brought to you for free and open access by the Jefferson Digital Commons. The Jefferson Digital Commons is a service of Thomas Jefferson University's Center for Teaching and Learning (CTL). The Commons is a showcase for Jefferson books and journals, peer-reviewed scholarly publications, unique historical collections from the University archives, and teaching tools. The Jefferson Digital Commons allows researchers and interested readers anywhere in the world to learn about and keep up to date with Jefferson scholarship. This article has been accepted for inclusion in Department of Emergency Medicine Faculty Papers by an authorized administrator of the Jefferson Digital Commons. For more information, please contact: JeffersonDigitalCommons@jefferson.edu. 


\section{Authors}

Xiaoliang Wang, Wayne B. Lau, Yue-Xing Yuan, Ya-Jing Wang, Wei Yi, Theodore A. Christopher, Bernard L. Lopez, Hui-Rong Liu, and Xin-Liang Ma 


\section{Methylglyoxal Increases Cardiomyocyte Ischemia/Reperfusion Injury via Glycative Inhibition of Thioredoxin Activity}

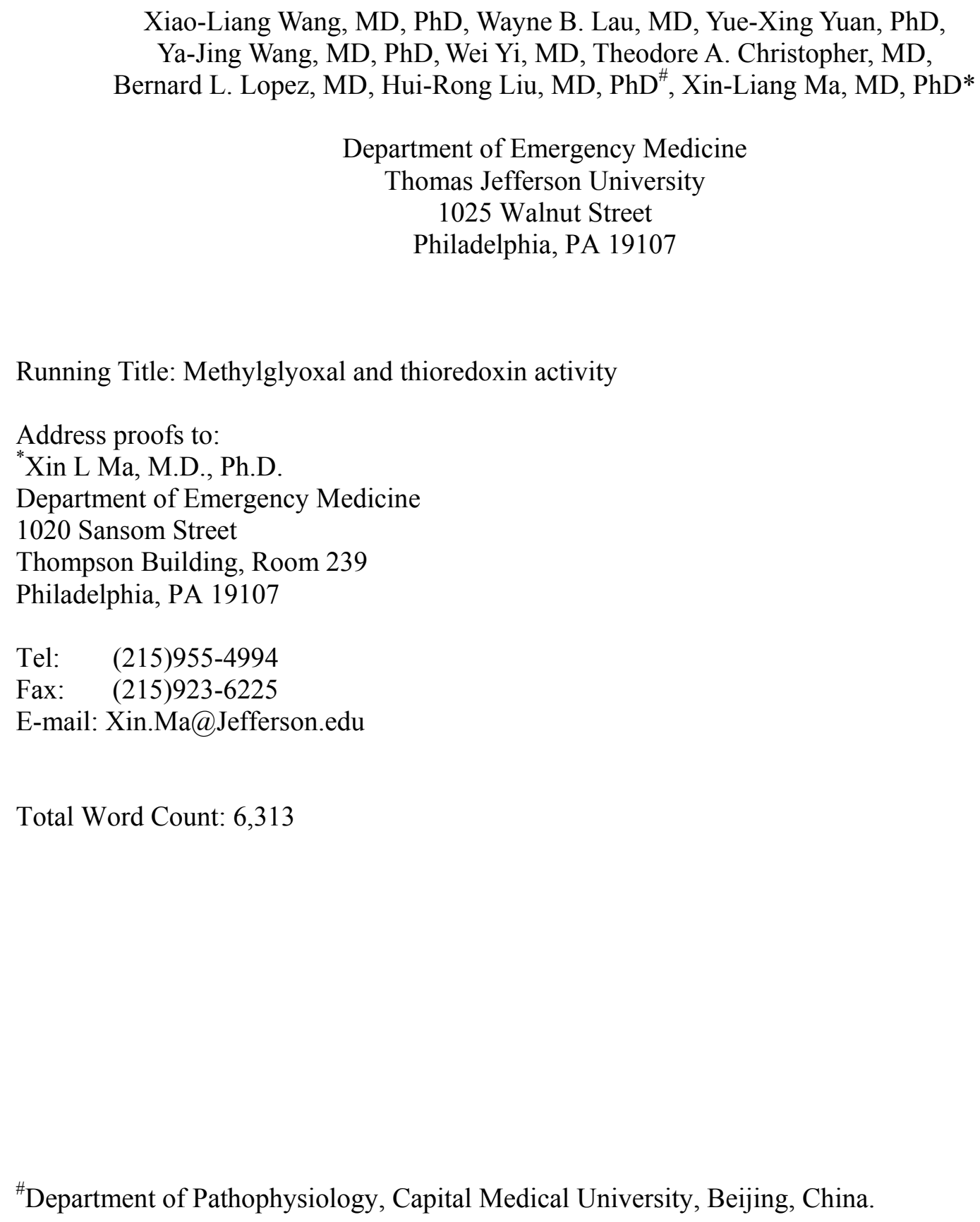


Abstract

Diabetes (DM) is closely related to cardiovascular morbidity and mortality, but the specific molecular basis linking DM with increased vulnerability to cardiovascular injury remains incompletely understood. Methylglyoxal (MG), a precursor to advanced glycation end-products (AGEs), is increased in diabetic patient plasma but its role in diabetic cardiovascular complications is unclear. Thioredoxin (Trx), a cytoprotective molecule with anti-apoptotic function, has been demonstrated to be vulnerable to glycative inhibition, but whether Trx is glycatively inhibited by MG thus contributing to increased cardiac injury has never been investigated. Cultured H9c2 cardiomyocytes were treated with MG $(200 \mu \mathrm{M})$ for 6 days. The following were determined pre- and post-simulated ischemia/reperfusion (SI/R) (8 hours hypoxia followed by 3 hours of reoxygenation): cardiomyocyte death/apoptosis, Trx expression and activity, AGEs formation, Trx-apoptosis-regulating kinase-1 (Trx-ASK1) complex formation, and p38 mitogen-activated protein kinase (MAPK) phosphorylation and activity. Compared to vehicle, MG significantly increased SI/R-induced cardiomyocyte LDH release and apoptosis $(P<0.01)$. Prior to SI/R, Trx activity was reduced in MG-treated cells, but 
Diabetes mellitus (DM) is a leading metabolic disorder in developed society, and causes devastating systemic consequences if poorly managed in the clinical setting. Considerable experimental and clinical data has demonstrated the close association between diabetes and significant cardiovascular morbidity and mortality. Recent studies have demonstrated that DM is a major risk factor for ischemic heart disease development (3), directly adversely affecting ischemic cardiomyocytes, resulting in larger infarct size and more severe heart failure after ischemia/reperfusion. Although many signaling pathways relating diabetic cellular injury and cardiac dysfunction have been reported, the specific molecular basis linking DM with increased vulnerability to ischemia/reperfusion injury and resultant mortality has not been established.

Methylglyoxal (MG), a highly reactive dicarbonyl, is a natural metabolite in glucose metabolism. It is capable of inducing the non-enzymatic reaction glycation, or glycosylation, between reducing sugars and proteins and other biomolecules, yielding irreversible advanced glycation end-products (AGEs) $(5 ; 28)$. The concentration of methylglyoxal is increased not only in diabetic animal tissues(37), but also in the plasma of diabetic patients(4;11). Elevated MG levels are believed to contribute to complications seen in poorly controlled diabetic states. Indeed, recent investigations have demonstrated that MG induces apoptosis of rat Schwann cells(12) and human vascular endothelial cells(2), buttressing evidence to the significant role MG plays in the etiology of diabetic complications. However, the role of methylglyoxal in ischemic injury endured in the diabetic cardiomyocyte, and any potentially involved mechanisms (apoptotic or otherwise) remain unidentified.

Thioredoxin (Trx), a 12-kDa protein ubiquitously expressed in all living cells, fulfills a variety of biological functions related to regulation of cellular proliferation and apoptosis(41), and cytoprotection against oxidative stress(46). Clinical and experimental results have demonstrated that inhibition of Trx promotes apoptosis(24). Recent in vitro studies 
104 demonstrate that Trx interacts directly with and inhibits the activity of apoptosis-regulating 105 kinase-1 (ASK1), a mitogen activated protein kinase (MAPK) that activates two proapoptotic 106 kinases, p38 MAPK and c-Jun N-terminal kinase (JNK)(26). These results give mechanistic 107 insight as to how Trx may critically regulate the balance between cell proliferation and cell 108 death

109 Recent studies have demonstrated that besides upregulation or downregulation of $\operatorname{Trx}$ 110 expression at the gene level, Trx activity is regulated by post-translational modification. Five 111 forms of post-translational modifications of Trx have been previously identified, each 112 modification affecting Trx differently. These include oxidation, glutathionylation, 113 S-nitrosylation, nitration, and glycation. We have recently demonstrated that Trx is susceptible 114 to non-enzymatic glycation via lipopolysaccharide (LPS) exposure (Free Radical Biology and 115 Medicine, in revision), consequent inactivation, and is furthermore unable to provide 116 protection against LPS-induced liver toxicity. However, whether Trx activity is altered in the 117 presence of prolonged methylglyoxal exposure, and any functional consequence of such 118 alteration with respect to cardiomyocyte protection against simulated ischemia/reperfusion has never been investigated.

120 Therefore, the aims of the present study were (1) to determine whether long-term 121 treatment with MG can enhance the injury of cultured H9c2 cardiomyocytes subjected to 122 simulated ischemia/reperfusion (SI/R), (2) if so, to investigate whether Trx-activity was 123 reduced after long-term treatment with $\mathrm{MG}$, (3) to determine the signaling mechanism(s) by 124 which reduced Trx activity leads to increased cardiomyocyte death after prolonged MG 125 exposure, and (4) to investigate whether administration of glycation inhibitor aminoguanidine 126 (AG) might be a therapeutic intervention reversing the observed phenomena related to 127 methylglyoxal exposure of cardiomyocytes. 
129 Material and methods

130 Cell culture and experimental protocol: $\mathrm{H} 9 \mathrm{c} 2$ cardiomyoblast cells (referred to as 131 cardiomyocytes thereafter), an embryonic rat heart-derived cell line (the American Type 132 Culture Collection, Manassas, VA), was cultured in Dulbecco's modified Eagle's medium 133 (DMEM; Invitrogen, Carlsbad, CA) containing 10\% calf bovine serum (CBS; MP Biomedical, 134 Solon, $\mathrm{OH})$, penicillin $(100 \mathrm{U} / \mathrm{ml})$, and streptomycin $(100 \mu \mathrm{g} / \mathrm{ml})$. The cells were maintained at $13537^{\circ} \mathrm{C}$ under a water-saturated atmosphere of $95 \%$ ambient air and $5 \% \mathrm{CO} 2$ (normoxic conditions). Stock cultures were passaged at 2- to 3-day intervals. Cells were seeded at a density of $3 \times 10^{5}$ cells per 35 -mm well of 6 -well plates for 24 -hour culture and were made quiescent by overnight serum-starvation (0\% CBS). After 6 days treatment with MG $(200 \mu \mathrm{M}$; Sigma-Aldrich, St. Louis, MO) or MG $(200 \mu \mathrm{M})$ + aminoguanidine hemisulfate (AG, $100 \mu \mathrm{M}$; Sigma-Aldrich, St. Louis, MO), the cells were subject to simulated ischemia/reperfusion (SI/R) as described previously(30). Briefly, the cells were incubated in slightly hypotonic Hanks' balanced saline solution $(1.3 \mathrm{mmCaCl} 2,5 \mathrm{~mm} \mathrm{KCl}, 0.3 \mathrm{mmKH} 2 \mathrm{PO} 4,0.5 \mathrm{~mm} \mathrm{MgCl} 2,0.4 \mathrm{~mm}$ MgSO4, $69 \mathrm{~mm} \mathrm{NaCl}, 4 \mathrm{~mm} \mathrm{NaHCO}$, and $0.3 \mathrm{mmNa} 2 \mathrm{HPO} 4)$ without glucose or serum, and transferred in an airtight incubator from which oxygen was removed and replaced by nitrogen for 8 hours at $37^{\circ} \mathrm{C}$. The incubator oxygen concentration $(1 \%)$ was adjusted to simulate 146 hypoxic conditions. Following hypoxia, the cells were re-oxygenated for 3 hours in DMEM with $1 \%$ serum at $37^{\circ} \mathrm{C}$. Sham-cells incubated in DMEM with $1 \%$ serum were not subjected to hypoxia. MG and AG were dissolved in double distilled $\mathrm{H} 2 \mathrm{O}$ (ddH2O). Cells received ddH2O as vehicle in control experiments. MG or AG was only present during the 6-day pre-incubation period, but not during SI/R period. Concentrations of MG and incubation time was established

151 based on dose and time-dependent pilot experiments.

152 Lactate dehydrogenase (LDH) activity assay: Post-treatment completion, all conditioned 153 medium was collected, and the cells were lysed(21). In short, a $100 \mu 1$ medium was added to 
$154100 \mu \mathrm{l}$ of a solution containing $100 \mathrm{mM}$ Tris buffer, $\mathrm{pH} 8.2,1.35 \mathrm{mM}$ tetrazolium salt, and 0.58 $\mathrm{mM}$ phenazine methosulphate, and $2.7 \mathrm{mM}$ NADH. The OD values were read at 0 and 30 minutes using a SpectraMax-Plus microplate spectrophotometer (Molecular Devices, Sunnyvale, CA) at $490 \mathrm{~nm}$. The percentage of $\mathrm{LDH}$ release was calculated as follows: $(\mathrm{A}-\mathrm{B}) /(\mathrm{C}-\mathrm{B}) \times 100$, where $\mathrm{A}=\mathrm{LDH}$ activity in conditioned media; $\mathrm{B}=\mathrm{LDH}$ activity in culture media (without cells); $\mathrm{C}=\mathrm{LDH}$ activity in cell lysates.

160 Assessment of cardiomyocyte apoptosis: Cardiomyocyte apoptosis was determined by terminal deoxynucleotidyl transferase-mediated dUTP nick end labeling (TUNEL) staining and caspase-3 activity, as we reported previously(41). TUNEL assay was performed utilizing the In Situ Cell Death Detection Kit (Roche, Palo Alto, CA). Briefly, cells were fixed with 10\% paraformaldehyde and incubated with the TUNEL reaction mixture containing TdT-mediated with IP Lab Imagine Analysis Software (version 3.5, Scanalytics, Fairfax, VA). Apoptotic index (number of TUNEL positively stained nuclei/total number of nuclei $\times 100$ ) was automatically calculated and exported for further analysis. Assays were performed in a blinded manner. The caspase-3 activity assay utilized the fluorogenic substrate DEVD-7-amino-4-trifluoromethyl-coumarin. Briefly, cells were lysed using caspase-3 lysis buffer (50mM HEPES PH 7.4, 0.1\% Chaps, 5mM DTT, 0.1 mM EDTA, 0.1\% Triton-X100), and total protein concentration was determined by the Bradford method. To each well of a 96-well plate, supernatant containing $50 \mu \mathrm{g}$ of protein was loaded and incubated with $3.645 \mu \mathrm{g}$ Ac-DEVD-AFC at $37^{\circ} \mathrm{C}$ for 1.5 hours. AFC was cleaved from DEVD by activated caspase-3, and the free AFC was quantified with a Biotek FL600 microplate fluorescence reader (excitation wavelength, $400 \mathrm{~nm}$; emission wavelength, $508 \mathrm{~nm}$ ). Caspase-3 activity was expressed as nanomoles of AFC formation per hour per milligram of protein. 
179 Trx activity assay: Trx activity was determined via the insulin disulfide reduction assay(17). In brief, $40 \mu \mathrm{g}$ of cellular protein extracts were pre-incubated at $37^{\circ} \mathrm{C}$ for 15 minutes with $2 \mu \mathrm{l}$ activation buffer (100 mM Hepes, $2 \mathrm{mMEDTA}, 1 \mathrm{mg} / \mathrm{ml} \mathrm{BSA}$, and $2 \mathrm{mM}$ dithiothreitol) to reduce thioredoxin. After addition of $20 \mu \mathrm{l}$ reaction buffer (100 mM Hepes, $2.0 \mathrm{mM}$ EDTA, 0.2 mM NADPH, and $140 \mu \mathrm{M}$ insulin), the reaction was initiated by addition of mammalian Trx reductase $\left(1 \mu \mathrm{l}, 15 \mathrm{mU}\right.$, Sigma), and samples were incubated for 30 minutes at $37^{\circ} \mathrm{C}$ (the controls received water only). The reaction was terminated by adding $125 \mu$ of stopping solution (0.2 M Tris-CL, $10 \mathrm{M}$ guanidine- $\mathrm{HCl}$, and 1.7mM 3-carboxy-4-nitrophenyl disulfide). Absorption measurement occurred at $412 \mathrm{~nm}$.

Immunocytochemical detection of advanced glycated end products: Cardiomyocytes, seeded on glass coverslips in 6 well plates, were treated as described above. Cells were fixed with polyformaldehyde (4\% in PBS) for 1 hour, washed with PBS, blocked with $10 \%$ normal goat serum, and incubated with rabbit anti-AGE polyclonal antibody (Abcam Inc., Cambridge, MA). Immunostaining was developed with a Vectastain $\mathrm{ABC}$ kit (Vector Laboratories, Burlingame, CA), and examined under light microscopy.

Detection of Trx-ASK1 interaction: Cells were homogenized with lysis buffer. Immunoprecipitation and immunoblotting were performed using a procedure described by Ischiropoulos and colleagues(43). In brief, endogenous Trx was immunoprecipitated with a monoclonal anti-murine Trx antibody (Redox Bioscience, Japan). After sample separation, the Trx-ASK-1 interaction was determined by Western blot analysis using a monoclonal antibody against ASK-1 (Upstate Biotechnology, Lake Placid, NY) and HRP-conjugated anti-mouse IgG antibody (Cell Signaling, Danvers, MA).

p38 MAPK activity assay: The p38 MAPK activity assay was performed utilizing a p38 MAPK assay kit (Cell Signaling Technology Inc., Danvers, MA) with substrate ATF-2, per manufacturer's instructions(14). In brief, cells were homogenized in ice-cold lysis buffer. 
204 Lysates were sonicated on ice, and centrifuged at $12,000 \mathrm{~g}$ for 10 minutes at $4^{\circ} \mathrm{C}$. 205 Immunoprecipitation was performed by adding $20 \mu \mathrm{l}$ of resuspended immobilized monoclonal 206 antibody against phospho-p38 MAPK (Thr180/Tyr 182) to $100 \mu$ cell lysate containing $150 \mu \mathrm{g}$ 207 of protein. The mixture was incubated with gentle rocking overnight at $4^{\circ} \mathrm{C}$. After $10,000 \mathrm{~g}$ 208 centrifugation at $4^{\circ} \mathrm{C}$ for 2 minutes, the pellets were washed twice with lysis buffer, and twice 209 with kinase buffer. The kinase reactions occurred in the presence of $200 \mu \mathrm{M}$ ATP and $2 \mu \mathrm{g}$ 210 ATF-2 fusion protein at $30^{\circ} \mathrm{C}$ for 30 minutes. After incubation, the samples were separated by SDS-PAGE, and ATF-2 phosphorylation was measured by Western immunoblotting using a 212 monoclonal antibody against phosphorylated ATF-2, followed by enhanced chemiluminescent 213 detection.

214 In vitro incubation of recombinant Trx with $M G$ or $M G+A G$ : To investigate the effects of $M G$ 215 on Trx directly, $1 \mu \mathrm{g}$ of recombinant human Trx (hTrx, Sigma-Aldrich, St. Louis, MO) was 216 incubated with $500 \mu \mathrm{M} \mathrm{MG}$ at $37^{\circ} \mathrm{C}$ for 8 days. Activity of treated $\operatorname{Trx}(0.3 \mu \mathrm{g})$ was then 217 determined as described above. Control Trx was incubated with deionized water under same 218 conditions. For the anti-glycation treatment, AG $(300 \mu \mathrm{M})$ was added at the initiation of 219 incubation.

220 Western blot analysis: Cultured Cardiomyocytes cells were collected in lysis buffer after 221 treatment. Aliquots containing 30-60 $\mu \mathrm{g}$ of protein were separated by electrophoresis through $2228-12 \%$ SDS-polyacrylamide gel, and transferred to positively charged nylon membranes. The membranes were blocked with $5 \%$ dry fat-free milk in Tris-buffered saline containing $0.1 \%$ 224 Tween 20 and then incubated with primary antibodies against Trx (Redox Bioscience, Japan), 225 p38, phospho-p38, GAPDH (Cell Signaling Technology Inc., Danvers, MA), and CML(Abcam 226 Inc., Cambridge, MA), respectively. Positively charged nylon membranes were then incubated 227 with horseradish peroxidase (HRP)-conjugated anti-rabbit immunoglobulin G antibody (Cell 228 Signaling, Danvers, MA) for 1 hour. The blot was developed with a Supersignal 
229 Chemiluminescence Detection Kit (Pierce, Rockford, IL). Bands were visualized with a Kodak

230 Images Station 400 (Rochester, NY), and the band densities were analyzed with Kodak

231 1-Dimensional software (version 3.6).

232 Statistical analysis: All values in the text, table, and figures are presented as means+SEM of $\mathrm{n}$ 233 independent experiments. All data (except western blot density) were subjected to ANOVA 234 followed by Bonferroni correction for post hoc $\mathrm{t}$ test. Western blot densities were analyzed 235 with the Kruskal-Wallis test followed by Dunn's post test. Probabilities of 0.05 or less were 236 considered to be statistically significant. 
$\underline{\text { Results }}$

Pre-culturing cardiomyocytes with high $M G$ increased their susceptibility to simulated ischemia/reperfusion injury. Cardiomyocytes subjected to SI/R injury manifested significant cellular injury including necrotic and apoptotic cell death, as evidenced by increased LDH release, TUNEL staining, and caspase-3 activity (Figure 1). Under basal conditions, cardiomyocytes treated with MG developed normally without apparent injury (MG+Sham SI/R groups in Figure 1). However, when subjected to SI/R, the cells treated with 6 days of MG $(200 \mu \mathrm{M})$ exhibited significantly greater cellular injury compared to control in the parameters listed above.

Pre-culturing cardiomyocytes with high MG caused significant protein glycation. As a highly reactive $\alpha$-oxoaldehyde, $\mathrm{MG}$ may modify proteins and other substrates via glycation. To determine whether pre-culturing cardiomyocytes with MG may cause cardiomyocyte protein glycation, AGE content in vehicle or MG-cultured cardiomyocytes was determined by immunohistological staining. As illustrated in Figure 2A and summarized in Figure 2B, exposure of cardiomyocytes to MG caused a concentration-dependent increase in AGE content with a 30.4-fold increase in AGEs formation observed at $200 \mu \mathrm{M}$ MG. Moreover, co-treatment of cardiomyocytes with aminoguanidine, a potent protein glycation inhibitor, markedly reduced high concentration MG-induced protein glycation (Figure 2, last bar).

Treatment with aminoguanidine protected $M G$-treated cardiomyocytes from simulated ischemia/reperfusion injury. Having demonstrated that pre-culturing cardiomyocytes with high concentration of MG significantly increased AGE formation and enhanced SI/R injury, we further determined whether increased protein glycation may play a causative role in enhanced cardiomyocyte SI/R injury. As summarized in Figure 3, co-treatment of cardiomyocytes with aminoguanidine, an advanced glycation end product formation inhibitor(33), significantly reduced LDH release (A), TUNEL staining (B), and caspase-3 activation (C) in high MG (200 
$262 \mu \mathrm{M})$ pre-treated cells subjected to $\mathrm{SI} / \mathrm{R}$. These results demonstrated that blockade of

263 MG-induced protein glycation significantly protected cardiomyocytes from SI/R injury.

$\underline{M G \text { incubation induced recombinant hTrx glycation and significantly decreased its activity. }}$

265 Our experimental results presented above demonstrated that MG is capable of initiating protein

266 glycation reactions and that blockade of protein glycation reduced SI/R injury in MG

267 pretreated cardiomyocytes. However, the specific proteins that are glycated by MG potentially

268 contributive to increased SI/R injury remain unknown. After a thorough literature review

269 identifying feasible protein candidates, Trx was selected because 1) Trx is a critical

270 anti-apoptotic and cell survival molecule, and its inactivation has been causatively related to

271 cardiovascular injury; and 2) we recently demonstrated the Trx is susceptible to glycative

272 modification by lipopolysaccharide and consequent activity inhibition. To directly test a novel

273 hypothesis that MG may cause Trx glycative inactivation, rendering cardiomyocytes more

274 susceptible to SI/R injury, we first determined whether Trx can be glycatively modified by MG

275 with subsequent activity inhibition. Recombinant hTrx (human thioredoxin-1) was incubated

276 with MG in a cell-free system, and Trx glycation and Trx activity were determined. As

277 summarized in Figure 4A, in vitro incubation of hTrx caused Trx glycation as evidenced by

278 abundant production of $\mathrm{N}^{\varepsilon}$-carboxymethyl lysine (CML), a biomarker of AGEs formation. MG

279 incubation markedly inhibited Trx activity (60.4\% reduction, $\mathrm{P}<0.01$ vs. vehicle-incubated

$280 \operatorname{Trx})($ Figure 4B). More importantly, addition of AG in the system completely abolished

281 MG-induced Trx glycation (Figure 4A) and significantly attenuated MG-induced Trx

282 inactivation (Figure 4B).

283 Trx is glycatively inhibited in $M G$ pre-treated cardiomyocytes prior to SI/R injury. Having

284 demonstrated that MG is capable of causing Trx glycative inhibition in a cell-free system, we

285 further determined whether cellular Trx activity/expression might be reduced after MG

286 exposure, leaving the cells more vulnerable to reperfusion injury. Compared to control, $\operatorname{Trx}$ 
activity was significantly decreased in the MG-treated cells prior to SI/R (Figure 5A). However, Trx expression was slightly increased in MG-treated cells (Figure 5B), indicating that the observed reduction in Trx activity in the MG-treated cells is not from reduced expression of the protein, but rather its posttranslational modification. More importantly, co-treatment with AG significantly attenuated MG inhibition of Trx (Figure 5A).

Pre-culturing cardiomyocytes with MG promoted SI/R-induced Trx-ASK1 dissociation and subsequent p38 MAPK activation, which were attenuated by co-treatment with AG. Recent in vitro studies have demonstrated that the binding and resultant inhibition of ASK1 is the primary mechanism by which Trx exerts its anti-apoptotic effect(38). Moreover, the increased ratio of ASK1/Trx-ASK1 correlates with the increased basal activity of the p38 MAPK pathway(18). To determine whether MG inhibition of Trx may alter the Trx-ASK1 interaction, and consequently activate downstream pro-apoptotic kinases, two additional experiments were performed. Via anti-Trx-1 immunoprecipitation and anti-ASK1 immunoblotting, Figure 6A illustrates Trx is physically associated with ASK1 in normal cultured cardiomyocytes, and this protein-protein interaction was significantly decreased after SI/R. Consequently, the activity of p38 MAPK, a pro-apoptotic downstream molecule for ASK1, was significantly enhanced in the MG-treated cardiomyocyte compared to control (Figure 6B). More importantly, this SI/R-induced disassociation of Trx-ASK1 was significantly further enhanced when cells were pre-cultured with MG, and p38 MAPK activity was significantly further increased (Figure 6). Treatment with aminoguanidine restored Trx-ASK1 interaction (Figure 7A), reduced p38 MAPK phosphorylation (Figure 7B), and inhibited p38 MAPK activity (Figure 7C) in MG-treated cardiomyocytes. 


\section{$\underline{\text { Discussion }}$}

Ischemic heart disease (IHD) continues to gain prevalence as a cause of disability and death in the United States, and is costly in terms of patient morbidity and mortality, as well as financial resources utilized in acute and chronic treatment. The specific molecular mechanisms underlying why diabetes mellitus directly increases IHD risk remain elusive. Accumulating evidence has indicated that $\mathrm{MG}$, a reactive dicarbonyl compound produced mainly from cellular glycolytic intermediates, is often found at high circulating blood levels in diabetic patients $(4 ; 11 ; 39)$. Evidence suggests elevated MG levels may play a role in the development of a number of diabetic complications(32). Elucidation of the effects of MG and other AGEs precursors upon the pre-ischemic heart, and the involved underlying mechanisms, could yield improved preventative and therapeutic treatment of the diabetic heart respectively at risk for and undergoing ischemic injury.

Our current study provided evidence that protein glycation is a new mechanism through which MG aggravates SI/R injury. This notion is supported by our observations that 1) pre-culturing cardiomyocytes with MG for 6 days caused a greater than 30 -fold increase in AGE production, which was dramatically reduced by co-treatment with AG, a strong AGE formation inhibitor; and 2) pre-culturing cardiomyocytes with MG for 6 days made cardiomyocytes more susceptible to SI/R as evidenced by increased LDH release, more cardiac caspase-3 activation, and greater percentage of TUNEL positive staining, all of which were also markedly inhibited by AG co-treatment. Two experimental limitations should be discussed. First, the MG concentration present in the culture medium is much higher than that found in diabetic patient plasma $(31 ; 35)$. However, it must be indicated that clinical situations are much more complicated, and actual MG concentrations to which in vivo cells are exposed remain uncertain. The intracellular MG level is likely much higher than the plasma MG level in the diabetic condition because diabetic tissues are chronically (months to years) exposed to high 
MG levels, which can cause dramatic intracellular MG accumulation (up to $300 \mu \mathrm{M})(7)$. In contrast, cultured cells were only transiently (days) exposed to high concentrations of MG, which may limit intracellular MG accumulation(8). Additionally, actual diabetic tissues are concomitantly exposed to high levels of plasma glucose, whereas cultured cells in this study were exposed to normal glucose concentration. Furthermore, as MG is formed during glycolysis, clinical diabetes, often causing tissue hypoperfusion and hypoxia, may stimulate intracellular MG production. In contrast, normal oxygen was present during the 6-day pre-culturing period of our study, and cellular glycolysis was minimal. For these reasons, high concentrations of MG ranging from $200 \mu \mathrm{M}$ to $1.5 \mathrm{mM}$ were typically used in previously published studies by many investigators $(10 ; 19 ; 23 ; 25 ; 45)$. Second, besides its strong anti-glycation property, AG is also a potent iNOS inhibitor. Therefore, the protective effect of AG against MG-enhanced SI/R injury could be attributed to its anti-iNOS effect. Although theoretically possible, our experimental results do not support this possibility because 1) we have previously demonstrated that significant iNOS upregulation begins 2 hours after reperfusion, but AG was washed out from the culturing system before the cells were subjected to SI/R; and 2) MG pre-culturing did not cause any significant cell injury (Figure 1, sham SI/R group) unless the cells were subjected to SI/R (Figure 1, SI/R group), and pre-treatment with AG alone during the pre-culturing period (washed out before SI/R) had no effect on cellular injury, before or after SI/R (data not shown).

Discovered 40 years ago in bacteria, thioredoxin's influence in human cells has only recently begun to be appreciated, as the diverse gamut of processes (including cellular redox balance, cell growth promotion, apoptosis inhibition, and inflammation modulation) regulated by thioredoxin continue to be discovered(34). It is not surprising, therefore, to behold the role Trx plays in a wide range of human diseases and conditions, including cancer, viral pathology, and ischemia/reperfusion injury(9). Emerging evidence suggests that Trx plays critical roles in 
360 promoting cell proliferation/survival and reducing cell death. Trx and its reductase protein 361 (TrxR) are upregulated in cancer tissues; molecules inhibiting Trx or TrxR promote apoptosis, 362 and reduce cancer development(36). In contrast, Trx activity is reduced in diseased tissues where pathologic apoptosis is increased(27). Recent studies have demonstrated that besides upregulation or downregulation of Trx expression at the gene level, Trx activity is differentially regulated by post-translational modifications. Oxidation of the thiol groups of Cys-32 and -35 forms a disulfide bond, and reversibly inhibits Trx's anti-oxidative activity. Glutathionylation, occurring at Cys-73, significantly inhibits Trx's antioxidant activity(6). S-nitrosylation occurs at Cys-69 or Cys-73, and has been shown to markedly enhance Trx's anti-oxidant, anti-apoptotic and organ protective activity $(15 ; 16 ; 41)$. Nitration, occurring at Ty-49, causes significant irreversible inhibition of Trx's anti-oxidative and cellular protection.

Protein glycation, also know as non-enzymatic glycosylation, is a protein modification reaction between proteins and reducing sugars(42). Glycation occurs in several steps. In an initial step that completes in a short period of time (minutes to hours), the reducing sugar reacts with the protein chain and produces Schiff-reaction primary-glycated products (e.g., fructosamine). After several days or weeks, Amadorial rearrangement commences, and advanced glycated end-products (AGEs) are formed(22). In recent years, the pathogenic roles of AGEs have been extensively investigated. Increased AGEs accumulation and subsequent tissue injury have been found in many human diseases, such as type 2 diabetes and the aging process $(1 ; 13 ; 44)$. However, whether the early modification of protein by sugar prior to AGEs formation may alter protein function remains largely unknown. A study by McCarthy et al(29) reported that incubation of alkaline phosphatase (ALP) with reducing sugars reduced enzyme activity associated with an increase in fructosamine levels, indicating that early glycation may alter protein function. In two more recent studies, it was reported that human $\mathrm{Cu}-\mathrm{Zn}$-superoxide dismutase(20) and esterase(40) can be glycated by methylglyoxal, and their 
activities are subsequently inhibited. Methylglyoxal reacts with the free amino groups of lysine and arginine, and with cysteine thiol groups to form AGEs. In a recent study, we have demonstrated that $\operatorname{Trx}$ is susceptible to non-enzymatic glycation via lipopolysaccharide (LPS) exposure (Free Radical Biology and Medicine, in revision), consequent inactivation, and is furthermore unable to provide protection against LPS-induced liver toxicity.

Our current study demonstrated for the first time that pre-culturing cardiomyocytes significantly inhibited cellular thioredoxin activity before the cells were subjected to SI/R, and caused greater dissociation of Trx-ASK1 and p38 MAPK activation after SI/R. The MG inhibition of Trx is likely attributable to $\operatorname{Trx}$ glycative modification and partially responsible for MG enhancement of SI/R injury. This novel hypothesis is supported by the following observations. Firstly, MG pre-culture slightly increased Trx expression, indicating that posttranslational modification, rather than Trx gene expression is responsible for reduced $\operatorname{Tr} x$ activity in MG pre-cultured cardiomyocytes (Figure 5). Secondly, MG caused significant recombinant hTrx-1 glycation and inactivation in a cell-free incubation system, and co-treatment with AG blocked Trx glycation and preserved Trx activity (Figure 4). Thirdly, addition of AG significantly attenuated the inhibitory effect of MG on cellular Trx activity (Figure 5). Finally, treatment with AG only during the pre-culturing period significantly improved Trx-ASK1 association and inhibited pro-apoptotic p38 MAPK activation after SI/R (Figure 7).

It should be indicated that Trx is also susceptible to nitrative inhibition. AG, as an iNOS inhibitor, may preserve Trx activity in MG-treated cells by blocking iNOS expression. However, our current study supports that Trx glycative modification is a more likely mechanism responsible for $\mathrm{MG}$ inactivation of $\operatorname{Trx}$ because 1) pre-culturing cells with $\mathrm{MG}$ significantly reduced Trx activity even before cells were subjected to SI/R, whereas significant iNOS expression was not observed until 2 hours after reperfusion; 2) treatment with AG only 
during the pre-culturing period where no significant iNOS is present significantly attenuated MG inactivation of Trx, and 3) in a cell-free system where no iNOS is present, AG blocked Trx glycation and preserved Trx activity after MG incubation.

Finally, some limitations should be addressed. Firstly, the specific amino acid residues of Trx-1 responsible for glycative modification remain unknown, and are currently under investigation. However, our preliminary data indicated that cysteine residues are not involved in glycative modification, because mutations of any or all of the $5 \operatorname{Trx}$ cysteine residues failed to block Trx-1 glycation. Secondly, we were unable to directly measure cardiomyocyte $\operatorname{Trx}$ glycation after MG incubation, because a method sensitive enough to detect early protein glycation in cells is currently unavailable. Nonetheless, our cell-free experimental results demonstrating that Trx function is glycatively inhibited, together with our cellular experimental results showing that cardiomyocyte Trx-1 activity is reduced in MG-treated cells and preserved by AG, summarily suggest that glycative Trx inactivation may contribute to MG enhancement of cardiomyocyte SI/R injury. Thirdly, H9c2 cells are neonatal myoblasts and may have some differences from the adult cardiomyocytes. However, this cell line has been extensively used as an experimental cardiomyocyte model, specially in those experiments that cells needs to be cultured for a long period of time.

In conclusion, our results demonstrated that thioredoxin activity was decreased due to posttranslational glycative modification in the cardiomyocytes treated with methylglyoxal. Blocking AGEs production inhibited Trx inactivation, and significantly protected the cardiomyocytes from SI/R injury. These results suggest that clinical therapeutic interventions preserving Trx activity or scavenging methylglyoxal in the diabetic setting may be novel modalities for attenuating injury endured in myocardial ischemia/reperfusion processes. 
434

\section{Acknowledgments}

435 This research was supported by the following grants: NIH HL-63828 and HL096686, 436 American Diabetes Association Research Award 7-08-RA-98, American Heart Association 437 Grant-in-Aid 0855554D (to X.L.M.), and NSFC 30700276 (to X.L.W)

438

439 Disclosures None 
1. Ahmed $\mathrm{N}$ and Thornalley PJ. Advanced glycation endproducts: what is their relevance to diabetic complications? Diabetes Obes Metab 9: 233-245, 2007.

2. Akhand AA, Hossain K, Mitsui H, Kato M, Miyata T, Inagi R, Du J, Takeda K, Kawamoto Y, Suzuki H, Kurokawa K and Nakashima I. Glyoxal and methylglyoxal trigger distinct signals for map family kinases and caspase activation in human endothelial cells. Free Radic Biol Med 31: 20-30, 2001.

3. Alpert JS. Diabetes mellitus and the risk for cardiovascular disease. Curr Cardiol Rep 5: 337, 2003.

4. Beisswenger PJ, Drummond KS, Nelson RG, Howell SK, Szwergold BS and Mauer M. Susceptibility to diabetic nephropathy is related to dicarbonyl and oxidative stress. Diabetes 54: 3274-3281, 2005.

5. Brownlee M. Lilly Lecture 1993. Glycation and diabetic complications. Diabetes 43: 836-841, 1994.

6. Casagrande S, Bonetto V, Fratelli M, Gianazza E, Eberini I, Massignan T, Salmona M, Chang G, Holmgren A and Ghezzi P. Glutathionylation of human thioredoxin: a possible crosstalk between the glutathione and thioredoxin systems. Proc Natl Acad Sci U S A 99: 9745-9749, 2002.

7. Chaplen FW, Fahl WE and Cameron DC. Evidence of high levels of methylglyoxal in cultured Chinese hamster ovary cells. Proc Natl Acad Sci U S A 95: 5533-5538, 1998.

8. Che W, Asahi M, Takahashi M, Kaneto H, Okado A, Higashiyama S and Taniguchi N. Selective induction of heparin-binding epidermal growth factor-like growth factor by 
methylglyoxal and 3-deoxyglucosone in rat aortic smooth muscle cells. The involvement of reactive oxygen species formation and a possible implication for atherogenesis in diabetes, J Biol Chem 272: 18453-18459, 1997.

9. Das DK. Thioredoxin regulation of ischemic preconditioning. Antioxid Redox Signal 6: 405-412, 2004.

10. Du J, Suzuki H, Nagase F, Akhand AA, Yokoyama T, Miyata T, Kurokawa K and Nakashima I. Methylglyoxal induces apoptosis in Jurkat leukemia T cells by activating c-Jun N-terminal kinase. J Cell Biochem 77: 333-344, 2000.

11. Fosmark DS, Torjesen PA, Kilhovd BK, Berg TJ, Sandvik L, Hanssen KF, Agardh CD and Agardh E. Increased serum levels of the specific advanced glycation end product methylglyoxal-derived hydroimidazolone are associated with retinopathy in patients with type 2 diabetes mellitus. Metabolism 55: 232-236, 2006.

12. Fukunaga M, Miyata S, Higo S, Hamada Y, Ueyama S and Kasuga M. Methylglyoxal induces apoptosis through oxidative stress-mediated activation of p38 mitogen-activated protein kinase in rat Schwann cells. Ann N Y Acad Sci 1043: 151-157, 2005.

13. Furber JD. Extracellular glycation crosslinks: prospects for removal. Rejuvenation Res 9: 274-278, 2006.

14. Gao F, Yue TL, Shi DW, Christopher TA, Lopez BL, Ohlstein EH, Barone FC and Ma XL. p38 MAPK inhibition reduces myocardial reperfusion injury via inhibition of endothelial adhesion molecule expression and blockade of PMN accumulation. Cardiovasc Res 53: 414-422, 2002.

15. Haendeler J, Hoffmann J, Tischler V, Berk BC, Zeiher AM and Dimmeler S. Redox 
regulatory and anti-apoptotic functions of thioredoxin depend on S-nitrosylation at cysteine 69. Nat Cell Biol 4: 743-749, 2002.

16. Haendeler J, Hoffmann J, Zeiher AM and Dimmeler S. Antioxidant effects of statins via S-nitrosylation and activation of thioredoxin in endothelial cells: a novel vasculoprotective function of statins. Circulation 110: 856-861, 2004.

17. Holmgren A and Bjornstedt M. Thioredoxin and thioredoxin reductase. Methods in Enzymology 252: 199-208, 1995.

18. Hsieh CC and Papaconstantinou J. Thioredoxin-ASK1 complex levels regulate ROS-mediated p38 MAPK pathway activity in livers of aged and long-lived Snell dwarf mice. FASEB J 20: 259-268, 2006.

19. Jia X, Olson DJ, Ross AR and Wu L. Structural and functional changes in human insulin induced by methylglyoxal. FASEB J 20: 1555-1557, 2006.

20. Kang JH. Modification and inactivation of human $\mathrm{Cu}, \mathrm{Zn}$-superoxide dismutase by methylglyoxal. Mol Cells 15: 194-199, 2003.

21. Korzeniewski C and Callewaert DM. An enzyme-release assay for natural cytotoxicity. $J$ Immunol Methods 64: 313-320, 1983.

22. Lapolla A, Traldi P and Fedele D. Importance of measuring products of non-enzymatic glycation of proteins. Clin Biochem 38: 103-115, 2005.

23. Li SY, Sigmon VK, Babcock SA and Ren J. Advanced glycation endproduct induces ROS accumulation, apoptosis, MAP kinase activation and nuclear O-GlcNAcylation in human cardiac myocytes. Life Sci 80: 1051-1056, 2007.

24. Lincoln DT, li Emadi EM, Tonissen KF and Clarke FM. The thioredoxin-thioredoxin 
reductase system: over-expression in human cancer. Anticancer Res 23: 2425-2433, 2003.

25. Liu BF, Miyata S, Hirota Y, Higo S, Miyazaki H, Fukunaga M, Hamada Y, Ueyama S, Muramoto O, Uriuhara A and Kasuga M. Methylglyoxal induces apoptosis through activation of 338 mitogen-activated protein kinase in rat mesangial cells. Kidney Int 63 : 947-957, 2003.

26. Liu Y and Min W. Thioredoxin promotes ASK1 ubiquitination and degradation to inhibit ASK1-mediated apoptosis in a redox activity-independent manner. Circ Res 90: 1259-1266, 2002.

27. Lovell MA, Xie C, Gabbita SP and Markesbery WR. Decreased thioredoxin and increased thioredoxin reductase levels in Alzheimer's disease brain. Free Radic Biol Med 28: 418-427, 2000.

28. Ma H, Li SY, Xu P, Babcock SA, Dolence EK, Brownlee M, Li J and Ren J. Advanced glycation endproduct (AGE) accumulation and AGE receptor (RAGE) up-regulation contribute to the onset of diabetic cardiomyopathy. J Cell Mol Med 13: 1751-1764, 2009.

29. McCarthy AD, Cortizo AM, Gimenez SG, Bruzzone L and Etcheverry SB. Non-enzymatic glycosylation of alkaline phosphatase alters its biological properties. $\mathrm{Mol}$ Cell Biochem 181: 63-69, 1998.

30. Mestril R, Chi SH, Sayen MR, O'Reilly K and Dillmann WH. Expression of inducible stress protein 70 in rat heart myogenic cells confers protection against simulated ischemia-induced injury. J Clin Invest 93: 759-767, 1994.

31. Mirza MA, Kandhro AJ, Memon SQ, Khuhawar MY and Arain R. Determination of glyoxal and methylglyoxal in the serum of diabetic patients by MEKC using 
stilbenediamine as derivatizing reagent. Electrophoresis 28: 3940-3947, 2007.

32. Monnier VM, Vishwanath V, Frank KE, Elmets CA, Dauchot P and Kohn RR. Relation between complications of type I diabetes mellitus and collagen-linked fluorescence. $N$ Engl J Med 314: 403-408, 1986.

33. Moriyama T, Kemi M, Okumura C, Yoshihara K and Horie T. Involvement of advanced glycation end-products, pentosidine and N(epsilon)-(carboxymethyl)lysine, in doxorubicin-induced cardiomyopathy in rats. Toxicology 268: 89-97, 2010.

34. Nakamura H. Thioredoxin as a key molecule in redox signaling. Antioxid Redox Signal 6: $15-17,2004$

35. Odani H, Shinzato T, Matsumoto Y, Usami J and Maeda K. Increase in three alpha,beta-dicarbonyl compound levels in human uremic plasma: specific in vivo determination of intermediates in advanced Maillard reaction. Biochem Biophys Res Commun 256: 89-93, 1999.

36. Powis G, Mustacich D and Coon A. The role of the redox protein thioredoxin in cell growth and cancer. Free Radic Biol Med 29: 312-322, 2000.

37. Randell EW, Vasdev S and Gill V. Measurement of methylglyoxal in rat tissues by electrospray ionization mass spectrometry and liquid chromatography. J Pharmacol Toxicol Methods 51: 153-157, 2005.

38. Saitoh M, Nishitoh H, Fujii M, Takeda K, Tobiume K, Sawada Y, Kawabata M, Miyazono K and Ichijo H. Mammalian thioredoxin is a direct inhibitor of apoptosis signal-regulating kinase (ASK) 1. EMBO J 17: 2596-2606, 1998.

39. Sell DR, Biemel KM, Reihl O, Lederer MO, Strauch CM and Monnier VM. Glucosepane 
40. Sen S, Bose T, Roy A and Chakraborti AS. Effect of non-enzymatic glycation on esterase activities of hemoglobin and myoglobin. Mol Cell Biochem 301: 251-257, 2007.

41. Tao L, Gao E, Bryan NS, Qu Y, Liu HR, Hu A, Christopher TA, Lopez BL, Yodoi J, Koch WJ, Feelisch M and Ma XL. Cardioprotective effects of thioredoxin in myocardial ischemia and reperfusion: Role of S-nitrosation. PNAS 101: 11471-11476, 2004.

42. Thorpe SR and Baynes JW. Maillard reaction products in tissue proteins: new products and new perspectives. Amino Acids 25: 275-281, 2003.

43. Vadseth C, Souza JM, Thomson L, Seagraves A, Nagaswami C, Scheiner T, Torbet J, Vilaire G, Bennett JS, Murciano JC, Muzykantov V, Penn MS, Hazen SL, Weisel JW and Ischiropoulos H. Pro-thrombotic state induced by post-translational modification of fibrinogen by reactive nitrogen species. J Biol Chem 279: 8820-8826, 2004.

44. Wolffenbuttel BH, Boulanger CM, Crijns FR, Huijberts MS, Poitevin P, Swennen GN, Vasan S, Egan JJ, Ulrich P, Cerami A and Levy BI. Breakers of advanced glycation end products restore large artery properties in experimental diabetes. Proc Natl Acad Sci U S A 95: 4630-4634, 1998.

45. Yamawaki H, Saito K, Okada M and Hara Y. Methylglyoxal mediates vascular inflammation via JNK and p38 in human endothelial cells. Am J Physiol Cell Physiol 295: C1510-C1517, 2008.

46. Yoshida T, Oka S, Masutani H, Nakamura H and Yodoi J. The role of thioredoxin in the aging process: involvement of oxidative stress. Antioxid Redox Signal 5: 563-570, 2003. 


\section{Figure Legends}

Figure 1. SI/R injury is significantly increased in $M G$ pre-cultured cardiomyocytes as measured by LDH release (A), apoptotic index by TUNEL positive staining cells (B), and caspase-3 activity (C). MG pre-culture results in cardiomyocyte glycation prior to being subject to $\mathrm{SI} / \mathrm{R}$ as measured by total AGEs content (D). $\mathrm{n}=6$ independent experiments. ${ }^{* *} \mathrm{P}<0.01$ vs. Sham $\mathrm{SI} / \mathrm{R},{ }^{\# \#} \mathrm{P}<0.01$ vs. vehicle $+\mathrm{SI} / \mathrm{R}$.

Figure 2. Effect of AG treatment on MG enhanced LDH release (A) and caspase-3 activity (B) after SI/R. $\mathrm{n}=5-6$ independent experiments.

Figure 3. Effect of AG treatment on MG enhanced apoptotic cell death determined by TUNEL positive staining after $\mathrm{SI} / \mathrm{R} . \mathrm{n}=5-6$ independent experiments.

Figure 4. Effects of MG on CML formation (A) and Trx activity of recombinant hTrx (B) in the absence and presence of $\mathrm{AG}$, an AGE formation inhibitor. $\mathrm{n}=6$ independent experiments. Effect of AG treatment on MG-exposed Trx inactivation (C) and Trx expression (D) in cardiomyocytes prior to being subject to SI/R. Insets: representative Western blots; bar graphs: density analysis ( $\mathrm{n}=5-8$ independent experiments).

Figure 5. MG pre-culture decreases Trx-ASK1 binding (A), and increases p38 MAPK activation (B). Insets: representative Western blots; bar graphs: density analysis $(\mathrm{n}=5-7$ independent experiments). Effect of AG treatment on MG enhanced Trx-ASK1 dissociation (C) and p38 MAPK phosphorylation (D) in cardiomyocytes subjected to SI/R. Insets: representative Western blots; bar graphs: density analysis ( $n=5-6$ independent experiments). 
A
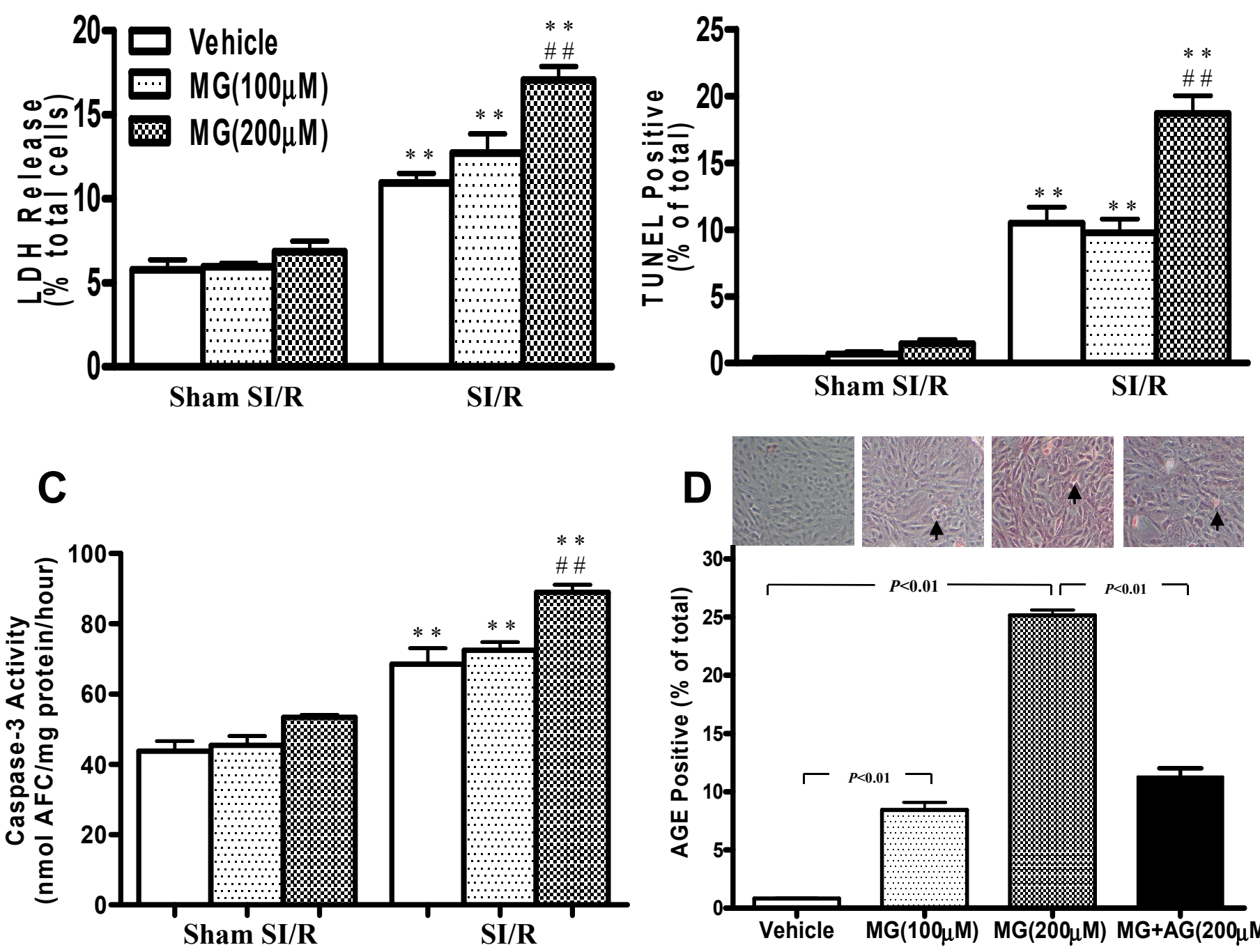

B

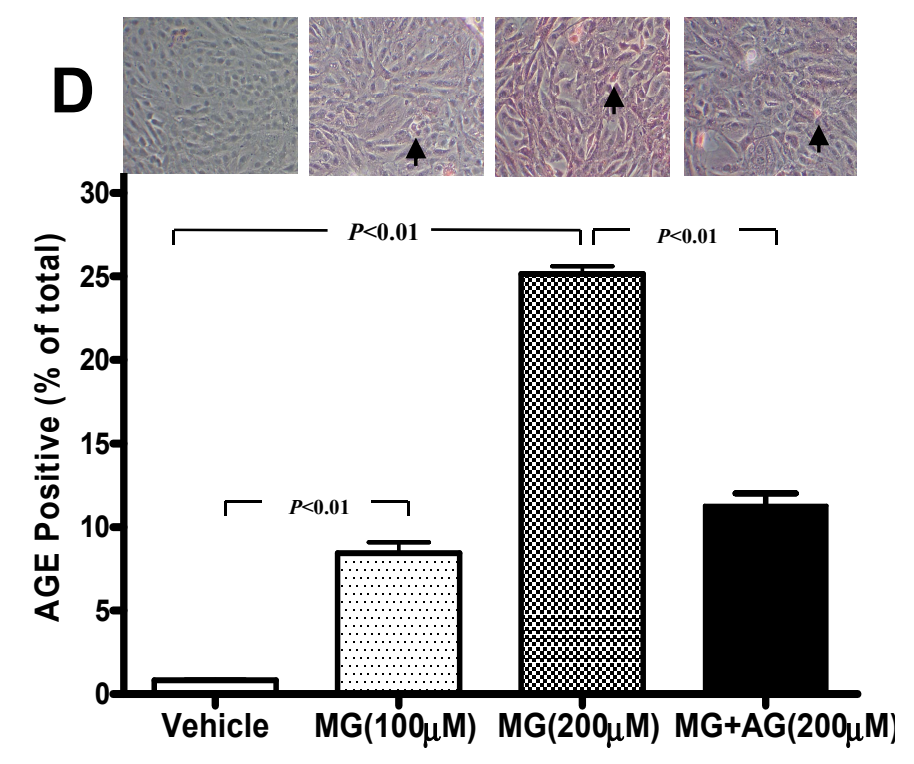

Figure 1 
A
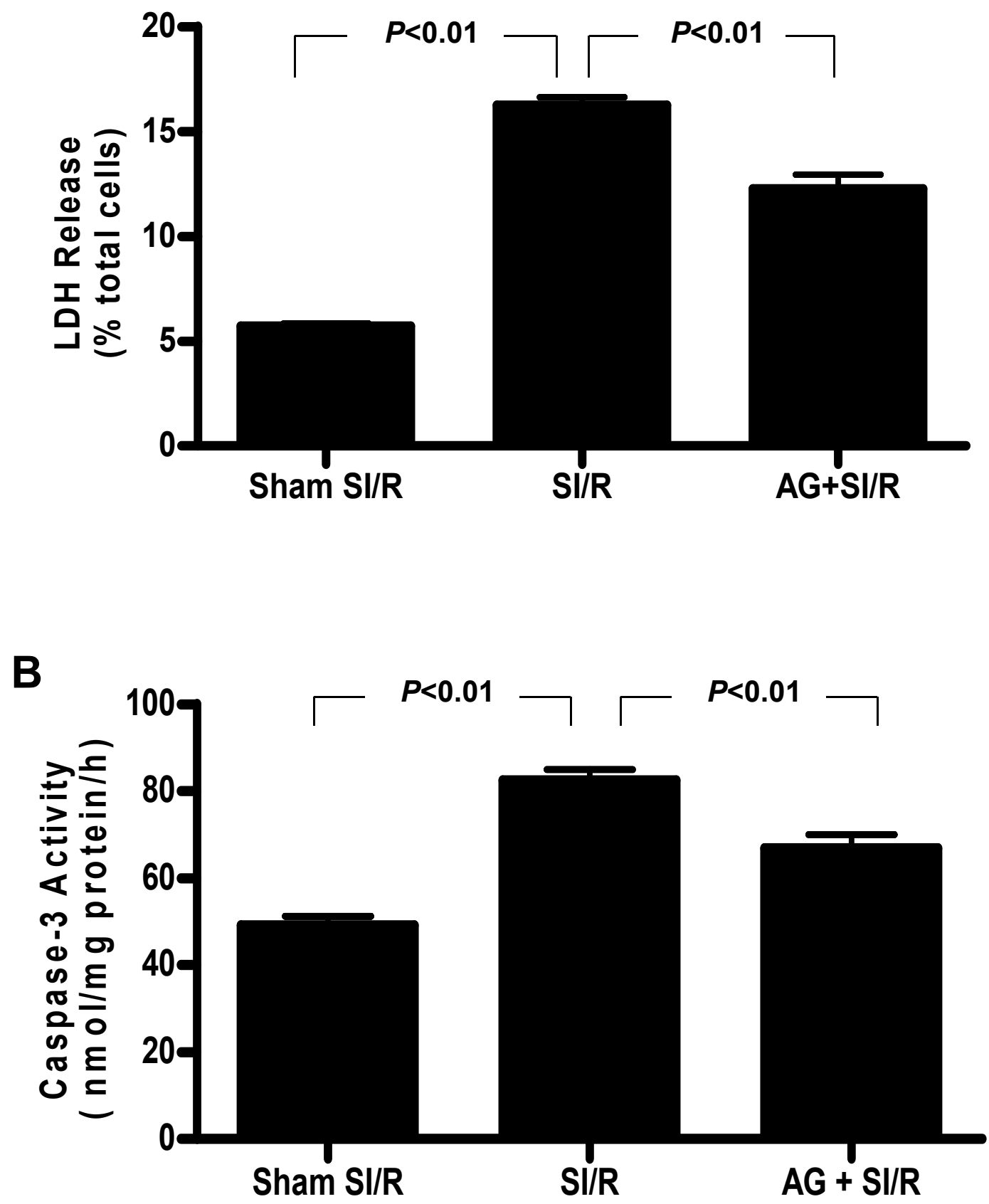

Figure 2 

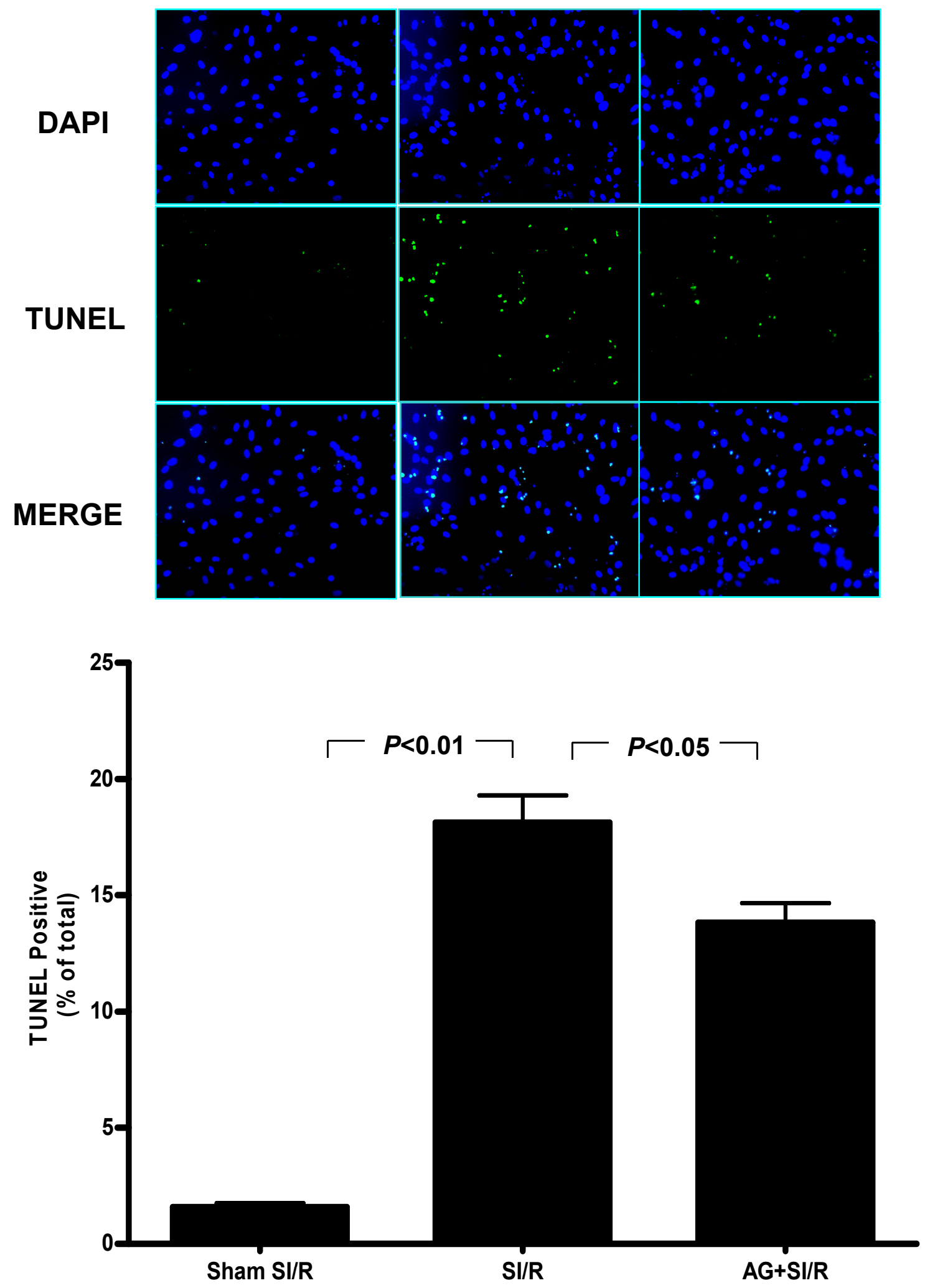

Figure 3 


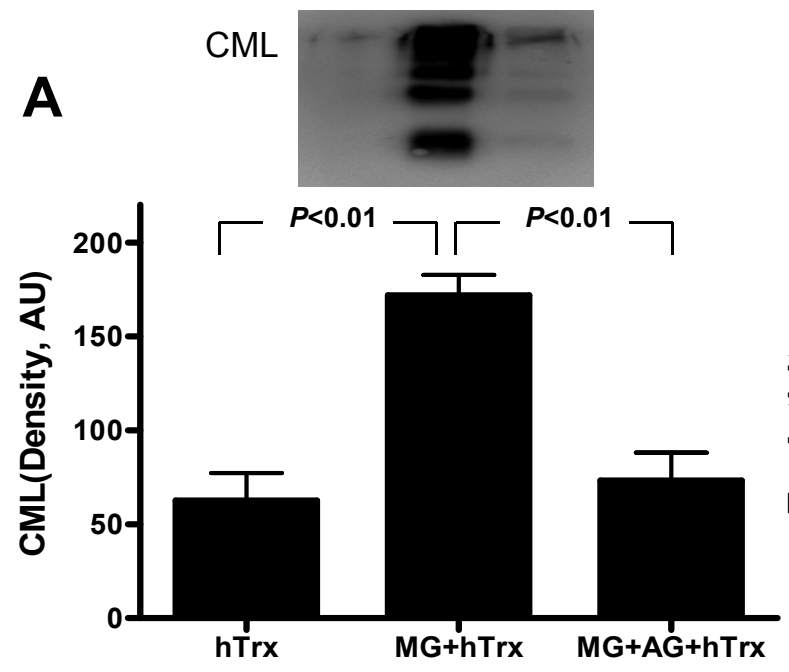

\section{B}

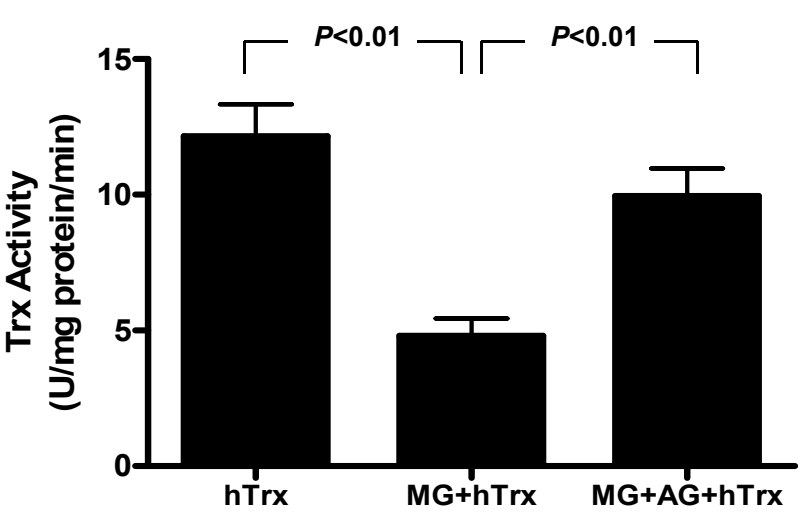

C
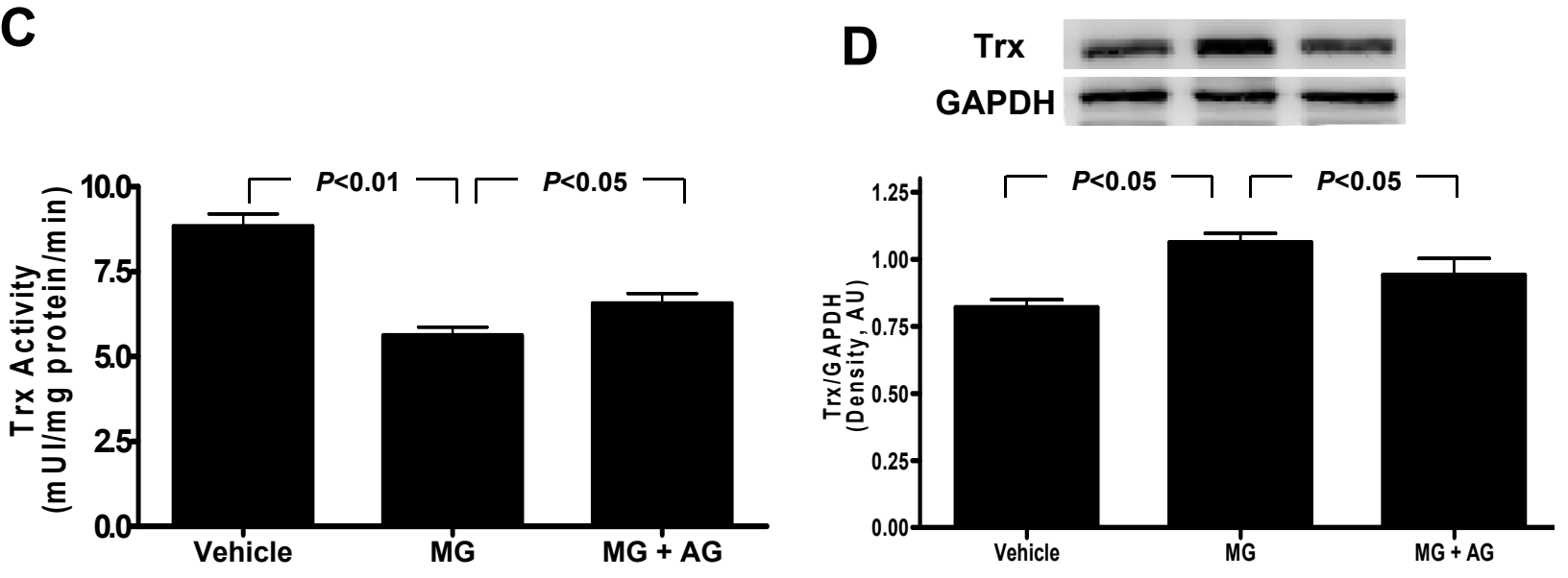

Figure 4 
A

IP: Trx

ASK1

Trx

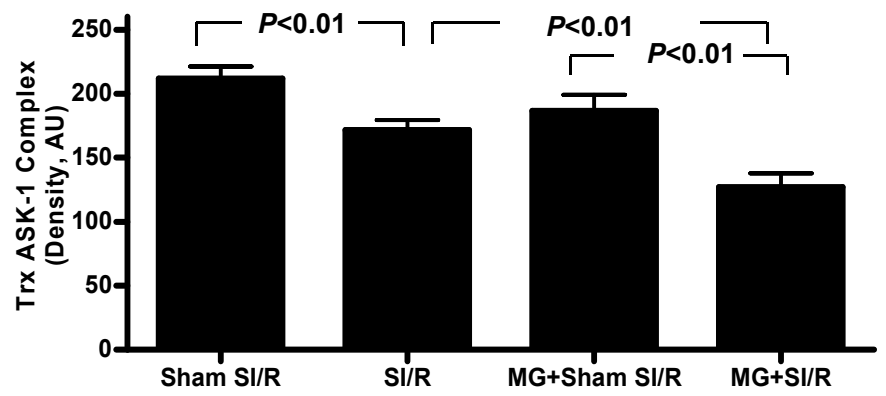

IP: Trx

C

ASK1

Trx

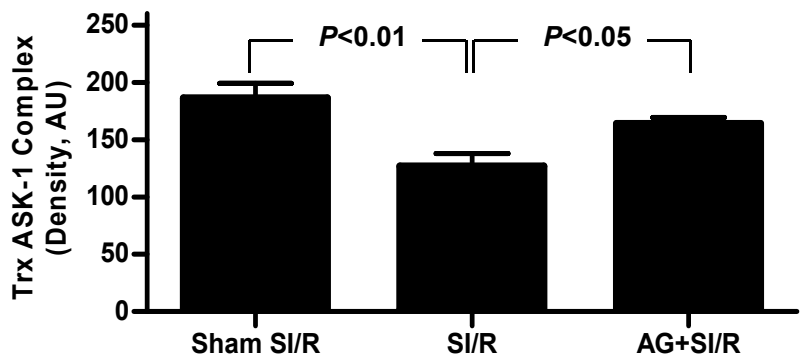

B

p-ATF2

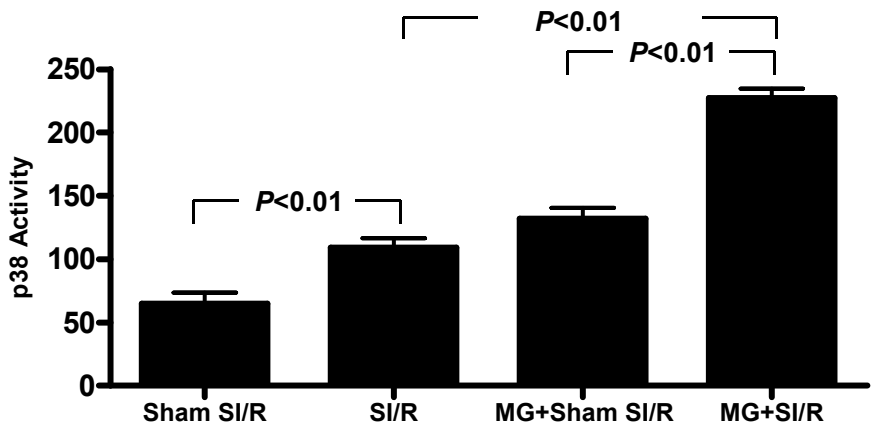

D Phospho-p38

p38

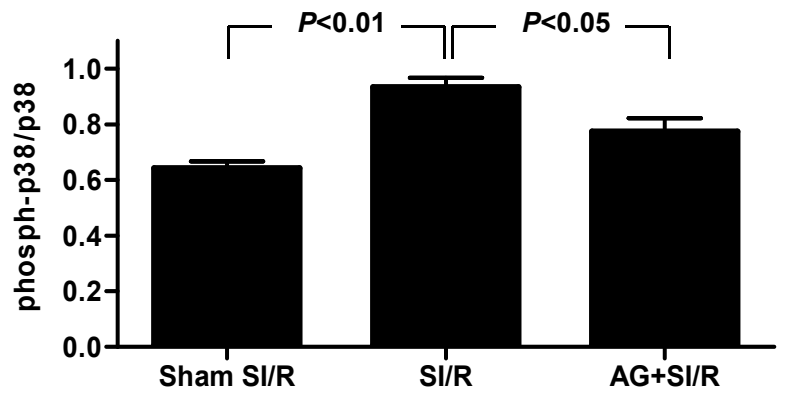

Figure 5 